\title{
Clinical relevance of elevated soluble ST2, HSP27 and 20S proteasome in patients with COVID-19
}

Ralph Wendt ${ }^{1}$ *, Marie-Therese Lingitz ${ }^{2,3 *}$, Maria Laggner ${ }^{2}$, Michael Mildner ${ }^{2,4}$ Denise Traxler $^{2,5}$, Alexandra Graf $^{6}$, Pavla Krotka ${ }^{6}$, Bernhard Moser ${ }^{2,7}$, Konrad Hoetzenecker ${ }^{7}$, Sven Kalbitz ${ }^{1}$, Christoph Lübbert ${ }^{1,8}$, Joachim Beige $^{1,9}$, Hendrik Jan Ankersmit ${ }^{2,7}$

* contributed equally

1 Department of Infectious Diseases, Tropical Medicine, Nephrology and Rheumatology, St. Georg Hospital, Delitzscher Str. 141, D-04129 Leipzig, Germany

2 Laboratory for Cardiac and Thoracic Diagnosis, Department of Surgery, Regeneration and Applied Immunology, Medical University of Vienna, Research Laboratories Vienna General Hospital, Waehringer Guertel 18-20, 1090 Vienna, Austria

3 Department of Anaesthesia, Intensive Care Medicine and Pain Medicine, Medical University of Vienna, Waehringer Guertel 18-20, 1090 Vienna, Austria

4 Department of Dermatology, Medical University of Vienna, Waehringer Guertel 18-20, 1090 Vienna, Austria 5 Division of Cardiology, Department of Internal Medicine II, Medical University of Vienna, Waehringer Guertel 18-20, 1090 Vienna, Austria

6 Center for Medical Statistics, Informatics and Intelligent Systems, Medical University of Vienna, Spitalg. 23, 1090 Vienna, Austria

7 Division of Thoracic Surgery, Department of Surgery, Medical University of Vienna, Waehringer Guertel 1820, 1090 Vienna, Austria

8 Division of Infectious Diseases and Tropical Medicine, Department of Medicine II, Leipzig University Medical Center, Liebigstr. 20, D-04103 Leipzig, Germany

9 Martin-Luther-University Halle-Wittenberg, Halle/Saale, Germany

Corresponding author:

HJ Ankersmit

Department of Thoracic Surgery, Medical University of Vienna

Waehringer Guertel 18-20

1090 Vienna, Austria

phone: $+43-(0) 1-40400-67770$

e-mail: hendrik.ankersmit@meduniwien.ac.at 


\begin{abstract}
Although, severe acute respiratory syndrome coronavirus - 2 (SARS-CoV-2) represents one of the biggest challenges in the world today, the exact immunopathogenic mechanism that leads to severe or critical Coronavirus Disease 2019 (COVID-19) has remained incompletely understood. Several studies have indicated that high systemic plasma levels of inflammatory cytokines result in the so-called "cytokine storm", with subsequent development of microthrombosis, disseminated intravascular coagulation, and multiorgan-failure. Therefore, we reasoned that elevated inflammatory cytokine might act as prognostic factors. Here, we analyzed 245 serum samples of patients with COVID-19, collected at hospital admission. We assessed the levels of heat shock protein 27 (HSP27), soluble suppressor of tumorigenicity- 2 (sST2), caspase cleaved cytokeratin 18 (cCK18), 20S proteasome, and tumor necrosis factor receptor 1 (TNFR-1) and explored their associations with overall-, 30-, 60-, 90-day- and in-hospital mortality. Moreover, we investigated their association with the risk of ventilation. We demonstrated that increased serum sST2 was uni- and multivariably associated with all endpoints. However, we also identified 20S proteasome as independent prognostic factor for in-hospital mortality. Furthermore, elevated HSP27, sST2, and 20S proteasome levels at hospital admission were univariably associated with higher risk of invasive ventilation. These findings could help to identify high-risk patients early in the course of COVID-19.
\end{abstract}




\section{Graphical Abstract:}

Figure 1 Graphical overview of the study.
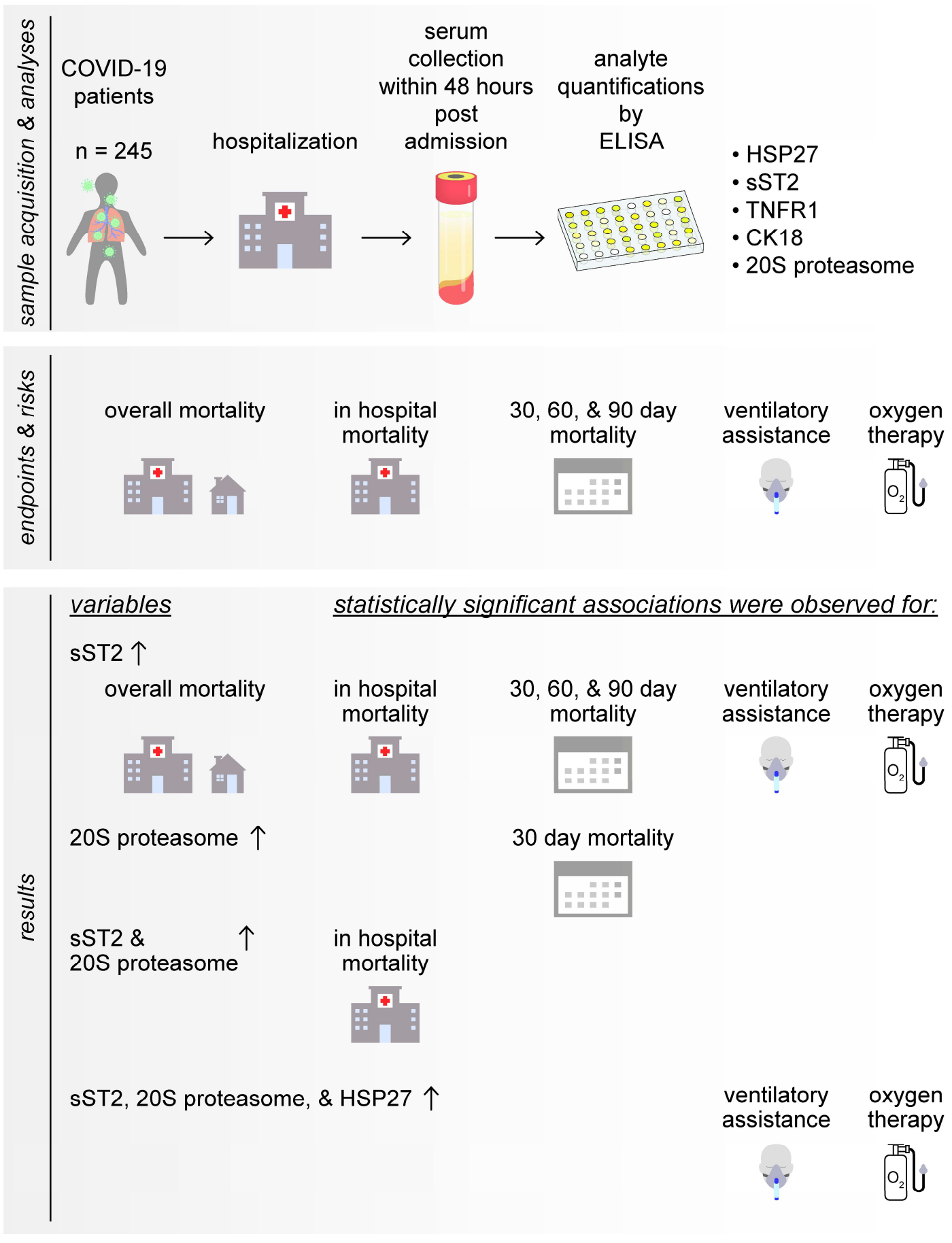

\section{Keywords}

COVID-19, HSP27, sST2, ARDS, biomarker 


\section{Introduction}

The severe acute respiratory syndrome coronavirus type 2 (SARS-CoV-2) pandemic is one of the largest challenges the world faces today. It remains difficult to estimate the frequencies of severe disease courses and fatalities linked to Coronavirus Disease 2019 (COVID-19), due to the number of undiagnosed and asymptomatic infections, which varies with differences in susceptibility and vulnerability among different populations. The reported hospitalization rates for patients with diagnosed SARS-CoV-2 infections varies between 7 and 25\% [1], [2]. The Robert Koch Institute reported 23\% mortality among patients hospitalized with COVID-19 in Germany [2]. Among patients that required intensive care in Germany, mortality rates were $30 \%$ and $36 \%$ for patients that received ventilation support [2]. Clinical deterioration or the development of severe or critical disease typically occurs later in the disease course, at least 7 to 10 days after the infection. Although several immunopathological mechanisms that lead to severe COVID-19 have been discovered, the exact immunopathogenesis remains incompletely understood. Several studies have indicated that pulmonary inflammation in COVID-19 was associated with high systemic plasma levels of inflammatory cytokines, which resulted in the so-called "cytokine storm" [3]-[6]. Inflammatory cytokines are released by infected cells, alveolar macrophages, recruited T lymphocytes, monocytes, and neutrophils. They increase the permeability of lung epithelium and reduce its barrier function. As a result, pulmonary edema fills the alveolar spaces, followed by the formation of hyaline membranes, similar to the early-phase features of acute respiratory distress syndrome (ARDS) [7], [8]. Indeed, cytokine overproduction is a characteristic feature of ARDS; consequently, several studies have suggested that a cytokine storm may be the main pathogenic factor in COVID-19-related ARDS. [9], [10]. Additionally, the overproduction of inflammatory cytokines is involved in the subsequent development of microthrombosis, disseminated intravascular coagulation, and multiorgan-failure. This imbalance between pro- and anti-coagulants was commonly found in patients that died of COVID-19, along with elevated d-dimer concentrations [11]-[13].

Indeed, COVID-19-associated ARDS displays several general characteristics of non-COVID-19-associated ARDS, such as impaired gas exchange and characteristic computed tomography (CT) findings. However, COVID-19 associated ARDS exhibits a more variable clinical appearance than non-COVID-19-ARDS [7], [14], [15]. Previous studies have also shown that reduced numbers of CD4+ and CD8+ T-cells were associated with severe COVID-19. That finding indicated that COVID-19 induced an immunodeficient state, comparable to the sepsis-induced immune-cell apoptosis observed in non-COVID-19-related ARDS and bacterial sepsis [3], [4], [8], [16]-[18].

Previous studies investigated immune-cell apoptosis in patients with sepsis [19]. They demonstrated that this phenomenon was associated with increased levels of soluble forms of circulating cluster of differentiation 95 (CD95), tumor necrosis factor 1 (TNF1), and interleukin-1-converting enzyme. Those findings suggested that lymphopenia, Th2 predominance, and subsequent immunodysfunction were caused by an aberrant state of T-cell activation that involved the CD95 pathway, the shedding of death-inducing receptors, and a tendency for CD4+ T-cells to undergo activation-induced cell death. Further studies [20], [21] implicated the involvement of elevated levels of $20 \mathrm{~S}$ proteasome and caspase cleaved cytokeratin 18 (cCK18) in immune-cell apoptosis. $20 \mathrm{~S}$ proteasome is a multi-catalytic proteinase complex involved in the enzymatic degradation of ubiquitinated proteins, and it serves as a marker of cell damage and immunological activity in autoimmune diseases. CCK18 is a marker of apoptosis that is not detectible in vital or necrotic cells, which indicates that cCK18 is associated with a higher apoptotic turnover during sepsis. In support of the hypothesis that, in sepsis, Th2 predominance 
arises from a shift from Th1 to Th2-biased cell proliferation, Brunner et al. [22] showed an increase in the levels of soluble suppressor of tumorigenicity (sST2), a member of the interleukin-33 (IL-33) family. Indeed, sST2 is predominantly expressed in type-2 T helper (Th2) cells, but not Th1 cells, which suggests that sST2 plays a role in cell proliferation and the promotion of the Th2 immune response. Additionally, Mildner et al [23] showed that sST2 was mainly produced in alveolar epithelial cells in the lung and cardiac myocytes. They also detected a strong augmentation of sST2 serum levels within $24 \mathrm{~h}$ post-LPS infusion, which implied a strong inflammatory response, accompanied by the secretion of inflammatory cytokines, such as IL- 6 and TNF- $\alpha$. Moreover, heatshock-protein 27 (HSP27), a member of the small heat shock protein family, was identified as an early marker of pulmonary damage and disease severity in patients with chronic obstructive pulmonary disease (COPD) [24], [25].

Considering these observations, we hypothesized that concentrations of the above-mentioned proteins might also be elevated in patients with severe COVID-19, and they might act as prognostic markers of survival. In the present study, we investigated the survival of 245 patients with COVID-19 and determined serum levels of sST2, TNF1 receptor (TNFR1), cCK18, HSP27, and 20S proteasome in specimens collected at hospital admission. We then explored which inflammatory markers were associated with a poorer prognosis in overall survival, 30-, 60and 90-day survival and in-hospital mortality. In addition, we investigated which parameters could indicate higher risk for patients requiring ventilatory assistance or oxygen therapy. 


\section{Materials and Methods}

\section{Study population}

From August 2020 to February 2021, serum samples were collected from patients infected with SARS-CoV-2 that were treated in the Department of Infectious Diseases and Tropical Medicine at St. Georg Hospital , in Leipzig. The treating unit included a standard hospital ward (33 beds) and an intermediate-care unit with 7 beds. At the time of admission, routine laboratory parameters were determined, including complete blood counts and C-reactive protein (CRP). In addition, laboratory parameters and clinical information, such as comorbidities and the course of disease, were derived from the medical history of each patient. Among the 270 serum samples, 25 were excluded, due to duplicate samples and patients with missing values. Thus, 245 patient samples were included in this study.

None of the patients had been vaccinated for SARS-CoV-2 prior to study inclusion. Patients that required extracorporeal membrane oxygenation procedures were transferred to another hospital, which was considered a discharge in our analyses.

All patients provided written informed consent prior to blood collection and participation.

Serum samples

Venous blood was drawn within $48 \mathrm{~h}$ of hospital admission. Whole blood was centrifuged at $4075 \mathrm{~g}$ for 4 minutes at room temperature. After centrifugation, aliquoted samples were stored at $-80^{\circ} \mathrm{C}$, until tests were performed.

Quantification of serum HSP27, TNFR1, sST2, cCK18, and 20S proteasome

We assessed serum levels of HSP27, sST2, and TNFR1 with commercially available ELISA kits (R\&D Systems, Minneapolis, Minnesota, USA), as described previously [26] [27] (Roth et al., 2004). The circulating soluble CK18 M30 neo-epitope was measured with commercially available ELISA kits from PEVIVA AB (Teco Medical, Sissach, Switzerland), as described previously [21] [28]. 20S proteasome serum levels were assessed with a commercial ELISA kit (Enzo Life Sciences, Farmingdale, New York, USA). All ELISA assays were performed according to the manufacturers' protocols. Colorimetric measurements were performed using Tecan F50 infinite microplate reader (Tecan Group, Männedorf, Switzerland) with Magellan software (version 7.2, Tecan). Analytes were quantified according to external standard curves.

\section{Statistical analysis}

Statistical analyses were performed using R, version 3.6.2. Data on 245 patients positive for SARS-CoV-2 were analyzed and associations between proinflammatory cytokines (HSP27, sST2, TNFR1, CK18, 20S proteasome) and the following endpoints were investigated: Overall mortality, 30-, 60- and 90-day-mortality, in-hospital mortality, requirement of ventilatory assistance or oxygen therapy.

For the analysis of overall mortality, uni- and multivariable Cox regression models were computed. Binary endpoints were analyzed using uni- and multivariable logistic regression as well as ROC curves. The results of the Cox regression models are presented using Hazard Ratios (HR) with corresponding 95\% Confidence Intervalls (CI) and for the binary endpoints, results of the logistic regression models are presented as Odds Ratios (OR) with 95\% CI. ROC curves and AUCs with 95\% CIs are presented. All possible predictors were at first assessed in an univariable analysis. Subsequently, multivariable models were computed including only 
significant predictors from the univariable analyses. Due to the exploratory character of the study, no correction for multiplicity was performed and $\mathrm{p}$-values $<0.05$ were considered statistically significant. 


\section{Results}

Study population characteristics

After excluding duplicate samples and patients with missing data, we included serum samples of 245 patients with SARS-CoV-2 infections. Demographic, clinicopathological, and laboratory details are shown in Table 1.

The study population displayed a female to male ratio of 46.1:53.9 with a median age of 76 years (IQR: 64-83). The majority of the study population comprised patients with pneumonic infiltrates, detected by thorax CT, and most $(n=137,55.9 \%)$ required oxygen supplementation at hospital admission, which corresponded to a WHO outcome classification of 4, based on the Ordinal Scale for Clinical Improvement [29]. The median follow-up time was 108 days (IQR: 19.5-162.5 days). The numbers of deaths after 30, 60, and 90 days were 44 (19.91\%), $52(23.64 \%)$, and $52(25.37 \%)$, respectively. The total number of deaths during follow up was 54 . Taken the $60-$ day post-hospital mortality, 60 days after discharge 13 from 180 patients died after initially surviving COVID-19 hospitalization. Twenty-six patients with shorter follow-up than 60 days and 39 patients that already died in the hospital were excluded for this analysis.

\section{Soluble ST2 serum content is significantly associated with worse overall survival}

To assess a potential prognostic impact of investigated inflammatory cytokines on Overall Survival, a univariable Cox-Regression model was performed. In our univariable analysis, age (measured in years) and sST2 (measured in $\mathrm{ng} / \mathrm{ml}$ ) are associated with worse overall survival in our study cohort $(\mathrm{HR}=1.364, \mathrm{p}=0.001$ ). Elevated sST2 levels remained significantly associated with higher mortality adjusting for age in the multivariable analysis $(\mathrm{HR}=1.353, \mathrm{p}=0.002)$. All other investigated inflammatory markers showed no significant association with worse overall survival. Detailed results are listed in Table 2.

Elevated sST2 acts as an potential prognostic factor for 30-, 60- and 90-days mortality, elevated 20S proteasome levels were associated with worse 30-days mortality

To investigate the association of assessed inflammatory markers with 30-, 60- and 90-day mortality, uni- and multivariable logistic regression models were computed. Since their follow up time was shorter than 30 days, 24 patients were excluded from the analysis. Within 30 days, 44 patients died. Increased levels of serum sST2 and $20 \mathrm{~S}$ proteasome were associated with higher 30 -days mortality in a univariable analysis (sST2: $\mathrm{OR}=1.536$, $\mathrm{p}<0.001 ; 20 \mathrm{~S}$ proteasome: $\mathrm{OR}=1.037, \mathrm{p}=0.004$ ). None of the other assessed biomarkers were significantly associated with worse prognosis in 30-days mortality. In multivariable analysis with inclusion of sST2, 20S proteasome and age, only sST2 remained significantly associated with the outcome (OR=1.457, $\mathrm{p}=0.004)$ (Table 3) ROC Curves and AUC values are depicted in Figure S1 and Table S1.

For 60-day mortality outcome, 25 patients were excluded since their follow up time was shorter than 60 days. Only sST2 was significantly associated with increased 60-day mortality risk (univariable: $\mathrm{OR}=1.335, \mathrm{p}=0.007$; multivariable: $1.298, \mathrm{p}=0.016$ ), whereas the other markers showed no significant effect on the 60-day outcome (Table 4). ROC curves and AUC are shown in Figure S2 and Table S2.

For the analysis of 90-day mortality, 40 patients were excluded due to shorter follow-up time. In our study cohort, 52 patients died during the first 90 days. In the univariable and multivariable logistic regression model, increased values of SST2 were significantly associated with higher 90-day mortality risk in both, the univariable $(\mathrm{OR}=1.335, \mathrm{p}=0.007)$ and multivariable $(\mathrm{OR}=1.304, \mathrm{p}=0.014)$ analyses. (Table 5) ROC Curves and AUC are presented in Figure S3 and Table S3. 
sST2 and 20S proteasome serum levels are significant predictors for in-hospital mortality

To evaluate the prognostic impact of investigated biomarkers on hospital survival, logistic regression models were performed. Of 245 included patients, 39 died in the hospital. In univariable analysis, sST2 (OR=1.697, $\mathrm{p}<0.001)$ and $20 \mathrm{~S}$ proteasome $(\mathrm{OR}=1.044, \mathrm{p}=0.019)$ were significantly associated with increased risk of inhospital mortality. This effects also remained in multivariable analysis $(O R=1.631$ and $1.041, p<0.001$ and 0.033 , respectively). The other investigated parameters showed no significant prognostic impact on in-hospital mortality. (Table 6) ROC Curves and AUC values are depicted in Figure S4 and Table S4.

Serum content of SST2, HSP27 and 20S proteasome might predict risk of intubation and requirement of oxygen therapy

We further evaluated the impact of inflammatory markers on endpoints such as the requirement of invasive ventilation or oxygen therapy. In our study cohort, 43 patients required invasive ventilation during hospitalization and oxygen therapy at hospital admission was needed in 179 cases. HSP27, sST2 and 20S proteasome serum levels were elevated in patients requiring invasive ventilation or oxygen therapy.

In the univariable analysis $\operatorname{HSP} 27(\mathrm{OR}=1.108, \mathrm{p}=0.041), \mathrm{sST} 2(\mathrm{OR}=1.79, \mathrm{p}<0.001)$ and $20 \mathrm{~S}$ proteasome $(\mathrm{OR}=1.039, \mathrm{p}=0.031)$ were identified as significant predictors for the risk of intubation. In the multivariable analysis, only sST2 $(\mathrm{OR}=1.709, \mathrm{p}<0.001)$ remained significantly associated with the outcome (Table 7).

HSP27 $(\mathrm{OR}=1.218, \mathrm{p}<0.001), \mathrm{sST} 2(\mathrm{OR}=1.258, \mathrm{p}=0.003)$ and $20 \mathrm{~S}$ proteasome $(\mathrm{OR}=1.177, \mathrm{p}<0.001)$ were significantly associated with the requirement of oxygen therapy at hospital admission in the univariable analysis. Increased values of these cytokines were also significantly associated with the need for oxygen therapy in the multivariable analysis $(\mathrm{OR}=1.134,1.175$ and $1.147, \mathrm{p}=0.037,0.046$ and 0.004 , respectively) (Table 8).

Correlations between sST2 with 20S proteasome, HSP27, and TNFR1

To identify potential correlations between the investigated biomarkers, we performed the Spearman rank correlation analyses. We found that ST2 was positively correlated with 20S proteasome, HSP27, and TNFR1 and that HSP27 was positively correlated with 20S proteasome (Table 9). 


\section{Discussion}

In this study, we examined the presence of soluble HSP27, sST2, TNFR1, cCK18, and 20S proteasome in serum samples obtained at hospital admission from patients with COVID-19. We confirmed previous studies which showed elevated sST2 levels in severe and fatal COVID-19 cases [30]-[32]. We also identified sST2 as an potential prognostic marker of overall mortality, 30-,60- and 90-day mortality, and in-hospital mortality. Serum sST2, 20S proteasome and HSP27 were also associated with the need of oxygen therapy or invasive ventilation. To our knowledge, this study was the first to examine HSP27 and 20S proteasome concentrations in SARS-CoV2 positive patients. Moreover, we showed that elevated serum sST2 and 20S proteasome levels at hospital admission were significantly associated with increased risk of in-hospital mortality in uni- and multivariable logistic regression.

sST2

ST2 is a member of the IL-1 receptor family. It is expressed in two main isoforms: a transmembrane cellular (ST2L) and a soluble (sST2) form. ST2 is the receptor for IL-33, which is secreted by living cells in response to cell damage [27], [33]. Alveolar epithelial cells in the lung and cardiac myocytes were previously identified as main sources of sST2 [23]. Because sST2 is inducible by inflammatory stimuli, it is elevated in a large number of diseases, including sepsis, trauma, burns, and heart failure [27], [34]-[36]. Another study assessed serum SST2 levels in patients with chronic heart failure [27] and showed that the median SST2 concentration was 3.631.ng/ml. In contrast, serum sST2 levels were greater than $400 \mathrm{ng} / \mathrm{ml}$ in patients on day 1 after a left ventricular assist device (LVAD) implantation, and subsequently, levels normalized by the end of the first postoperative week [37]. In comparison, the median SST2 value in our study cohort was $7.853 \mathrm{ng} / \mathrm{ml}$ (IQR: 6.584-8.935). Therefore, the median serum sST2 levels in patients with COVID-19 were twice as high as the levels found in patients with chronic heart failure. This finding suggested that patients with COVID-19 infections had undergone a remarkable production of inflammatory cytokines by the time they were admitted to the hospital. Moreover, we found SST2 as a potential prognostic factor for in-hospital mortality, 30-, 60- and 90day mortality. This finding supported the hypothesis that patients with severe COVID-19 exhibited strong inflammatory activation [5], [6], [8], and it confirmed previous findings that identified sST2 as a marker that clearly distinguished between patients with increased risk and patients with low risk of mortality [5], [6], [8], [38].

HSP27

HSP27 acts as an intracellular chaperone to maintain normal cell functions, but it is also active extracellularly. HSPs are involved in apoptosis inhibition, cytoprotection, and immunomodulation [39]-[41]. Moreover, HSP27 may play a major role in platelet aggregation by modifying actin polymerization [10], [42]. Tian et al. [10] found an association between a high thrombus burden and elevated HSP27 levels in patients with ST elevation myocardial infarctions. In addition, recently, HSP27 was identified as prognostic marker in inflammatory diseases, such as COPD and trauma-related ARDS [24], [25], [43], [44]. Therefore, we hypothesized that, in patients with COVID-19, elevated HSP27 might be associated with lung injury. Furthermore, the increase in HSP27 might have been related to thromboinflammation, which is the coordinated activation of inflammatory and thrombotic responses. Indeed, this condition might explain some of the key aspects of morbidity and mortality observed in patients with COVID-19 [10], [45]. 
HSP27 serum levels were previously assessed in healthy volunteers, who exhibited a median concentration of $1.482 \mathrm{ng} / \mathrm{ml}$ [46]. In our study cohort, the median HSP27 level at hospital admission was $5.839 \mathrm{ng} / \mathrm{ml}$ (IQR: 4.070-8.316), which was about four-fold higher than the levels found in healthy controls. In our study cohort, HSP27 was significantly associated with increased risk for invasive ventilation or the need of oxygen therapy, which could indicate an extensive damage of the lung in these patients.

\section{S proteasome}

Roth et al. [20] previously described elevated core $20 \mathrm{~S}$ proteasome levels in critically ill patients, particularly those with sepsis. Core $20 \mathrm{~S}$ proteasome is a marker of increased apoptotic turnover. Therefore, we investigated whether 20S proteasome levels could act as a prognostic marker for overall and in-hospital mortality. Surprisingly, we found that increased 20S proteasome levels were not significantly associated with overall-, 30,60- and 90-day mortality, but we found that $20 \mathrm{~S}$ proteasome was a significant predictor for the risk of invasive ventilation or the need of oxygen therapy. Increased $20 \mathrm{~S}$ serum proteasome was also significantly associated with higher in-hospital mortality risk. Elevated 20S proteasome was found in several other pathological conditions such as carcinoma [47], burn injuries[48] and autoimmune diseases [49]. These observations confirm our findings that patients in our study cohort with elevated 20S proteasome levels suffer from severe tissue damage and hyper-activated immune system, whereas these factors together lead to increased apoptosis and thus probably higher risk of invasive ventilation or higher in-hospital mortality [50].

\section{Survival}

Our population comprised patients primarily hospitalized in a non-intensive care unit (ICU) setting. Patients with severe diseases, up to WHO scale 5 (high-flow oxygen therapy), could be treated outside the ICU. Patients that required non-invasive or invasive ventilation had to be transferred to the ICU. Our cohort had a mortality of $19.91 \%$ after 30 days. However, the mortality of our study cohort increased to 23,64\% after 90 days, respectively. Additionally, the fact that after discharge from the hospital, nearly $8 \%$ of patients died after initially surviving COVID-19, reflect the possibly longer-term effects of the SARS-CoV-2 infection.

The main limitation of this study was the lack of a matched reference group without a COVID-19 history, for survival estimations in this vulnerable group of older patients (median age 76 years). However, our survival results suggested that COVID-19 also had an impact on survival after successful treatment of the acute infection and clearance of the virological disease.

\section{Conclusion}

With this study we evidenced that high level of sST2 could serve as prognostic marker for disease severity and overall survival. We demonstrated that serum sST2 was an early prognostic marker for overall mortality, 30-, 60and 90 day mortality and in-hospital mortality, and the need for invasive ventilation or oxygen therapy. Moreover, we showed that besides sST2, also 20S proteasome, and HSP27 were significantly associated with a subsequent requirement for invasive ventilation during hospitalization. In addition, we evidenced that the augmented release of these inflammatory cytokines was associated with the need for oxygen therapy at hospital admission. We also detected a significant increment in 20S proteasome and sST2 levels in patients that died during the hospital stay. These findings contributed to a broader understanding of the systemic inflammation response in patients with COVID-19 and might help identify high risk patients at admission. 


\section{Tables}

Table 1: Demographic characteristics and WHO outcome classification codes for the study population at hospital admission

\begin{tabular}{|c|c|c|c|c|c|c|c|c|c|}
\hline \multirow[b]{2}{*}{ Characteristic } & \multirow[b]{2}{*}{ All patients $(n=245)$} & & \multirow{2}{*}{\multicolumn{2}{|c|}{ Yes $(n=52)$}} & & \\
\hline & & Yes $(n=44)$ & No $(n=177)$ & & & Yes $(n=52)$ & No $(n=153)$ & Yes $(n=39)$ & No $(n=206)$ \\
\hline Age (years) & $76(64-83)$ & $80.5(74-86)$ & $74(62-83)$ & $80(74-86)$ & $74(62-83)$ & $80(74-86)$ & $74(62-83)$ & $81(74-86)$ & $74(62.25-82)$ \\
\hline \multicolumn{10}{|l|}{ Sex } \\
\hline Female & $113(46.12 \%)$ & $16(36.36 \%)$ & $83(46.89 \%)$ & $19(36.54 \%)$ & $80(47.62 \%)$ & $19(36.54 \%)$ & $69(45.1 \%)$ & $16(41.03 \%)$ & $97(47.09 \%)$ \\
\hline Male & $132(53.88 \%)$ & $28(63.64 \%)$ & $94(53.11 \%)$ & $33(63.46 \%)$ & $88(52.38 \%)$ & $33(63.46 \%)$ & $84(54.9 \%)$ & $23(58.97 \%)$ & $109(52.91 \%)$ \\
\hline \multicolumn{10}{|c|}{$\begin{array}{l}\text { WHO Outcome Classification } \\
\text { at admission }\end{array}$} \\
\hline 2 & $3(1.22 \%)$ & $0(0 \%)$ & $3(1.69 \%)$ & $0(0 \%)$ & $3(1.79 \%)$ & $0(0 \%)$ & $2(1.31 \%)$ & $0(0 \%)$ & $3(1.46 \%)$ \\
\hline 3 & $63(25.71 \%)$ & $8(18.18 \%)$ & $50(28.25 \%)$ & $11(21.15 \%)$ & $47(27.98 \%)$ & $11(21.15 \%)$ & $43(28.1 \%)$ & $7(17.95 \%)$ & $56(27.18 \%)$ \\
\hline 4 & $137(55.92 \%)$ & $22(50 \%)$ & $100(56.5 \%)$ & $26(50 \%)$ & $96(57.14 \%)$ & $26(50 \%)$ & $86(56.21 \%)$ & $18(46.15 \%)$ & $119(57.77 \%)$ \\
\hline 5 & $41(16.73 \%)$ & $13(29.55 \%)$ & $24(13.56 \%)$ & $14(26.92 \%)$ & $22(13.1 \%)$ & $14(26.92 \%)$ & $22(14.38 \%)$ & $13(33.33 \%)$ & $28(13.59 \%)$ \\
\hline 6 & $1(0.41 \%)$ & $1(2.27 \%)$ & $0(0 \%)$ & $1(1.92 \%)$ & $0(0 \%)$ & $1(1.92 \%)$ & $0(0 \%)$ & $1(2.56 \%)$ & $0(0 \%)$ \\
\hline \multicolumn{10}{|l|}{ at discharge } \\
\hline 0 & $26(10.61 \%)$ & $0(0 \%)$ & $19(10.73 \%)$ & $0(0 \%)$ & $19(11.31 \%)$ & $0(0 \%)$ & $19(12.42 \%)$ & $0(0 \%)$ & $26(12.62 \%)$ \\
\hline 1 & $14(5.71 \%)$ & $2(4.55 \%)$ & $11(6.21 \%)$ & $3(5.77 \%)$ & $10(5.95 \%)$ & $3(5.77 \%)$ & $9(5.88 \%)$ & $1(2.56 \%)$ & $13(6.31 \%)$ \\
\hline 2 & $131(53.47 \%)$ & $2(4.55 \%)$ & $115(64.97 \%)$ & $5(9.62 \%)$ & $111(66.07 \%)$ & $5(9.62 \%)$ & $105(68.63 \%)$ & $0(0 \%)$ & $131(63.59 \%)$ \\
\hline 3 & $18(7.35 \%)$ & $0(0 \%)$ & $17(9.6 \%)$ & $0(0 \%)$ & $17(10.12 \%)$ & $0(0 \%)$ & $13(8.5 \%)$ & $0(0 \%)$ & $18(8.74 \%)$ \\
\hline 4 & $12(4.9 \%)$ & $1(2.27 \%)$ & $10(5.65 \%)$ & $1(1.92 \%)$ & $10(5.95 \%)$ & $1(1.92 \%)$ & $7(4.58 \%)$ & $1(2.56 \%)$ & $11(5.34 \%)$ \\
\hline 5 & $1(0.41 \%)$ & $0(0 \%)$ & $1(0.56 \%)$ & $1(1.92 \%)$ & $0(0 \%)$ & $1(1.92 \%)$ & $0(0 \%)$ & $0(0 \%)$ & $1(0.49 \%)$ \\
\hline 7 & $1(0.41 \%)$ & $0(0 \%)$ & $1(0.56 \%)$ & $0(0 \%)$ & $1(0.6 \%)$ & $0(0 \%)$ & $0(0 \%)$ & $0(0 \%)$ & $1(0.49 \%)$ \\
\hline 8 & $42(17.14 \%)$ & $39(88.64 \%)$ & $3(1.69 \%)$ & $42(80.77 \%)$ & $0(0 \%)$ & $42(80.77 \%)$ & $0(0 \%)$ & 37 (94.87\%) & $5(2.43 \%)$ \\
\hline
\end{tabular}

Values are the median (interquartile range) or the number (\%), as indicated 
Table 2 Univariable and multivariable Cox-Regression in association with overall survival $(\mathrm{n}=245)$

\begin{tabular}{lllll}
\hline \hline & Hazard Ratio & Lower CL & Upper CL & p-Value \\
\hline Univariable Cox-Regression & & & & \\
Age & $\mathbf{1 . 0 4 7}$ & $\mathbf{1 . 0 2}$ & $\mathbf{1 . 0 7 4}$ & $<\mathbf{0 . 0 0 1}$ \\
HSP27 & 1.083 & 0.998 & 1.176 & 0.055 \\
sST2 & $\mathbf{1 . 3 6 4}$ & $\mathbf{1 . 1 3}$ & $\mathbf{1 . 6 4 7}$ & $\mathbf{0 . 0 0 1}$ \\
TNFR1 & 1.053 & 0.98 & 1.132 & 0.162 \\
cCK18 & 0.595 & 0.13 & 2.735 & 0.505 \\
20S proteasome & 1.019 & 0.998 & 1.041 & 0.076 \\
& & & & \\
Multivariable Cox-Regression & & & & $\mathbf{0 . 0 0 1}$ \\
Age & $\mathbf{1 . 0 4 7}$ & $\mathbf{1 . 0 1 9}$ & $\mathbf{1 . 0 7 5}$ & $\mathbf{0 . 0 0 2}$ \\
sST2 & $\mathbf{1 . 3 5 3}$ & $\mathbf{1 . 1 1 6}$ & $\mathbf{1 . 6 3 9}$ & \\
\hline
\end{tabular}


Table 3. Univariable and multivariable logistic regression in association with 30 -day mortality $(n=221)$

\begin{tabular}{lllll}
\hline \hline & Odds Ratio & Lower CL & Upper CL & p-Value \\
\hline Univariable Logistic Regression & & & & \\
Age & $\mathbf{1 . 0 5 6}$ & $\mathbf{1 . 0 2 3}$ & $\mathbf{1 . 0 9 4}$ & $\mathbf{0 . 0 0 1}$ \\
HSP27 & 1.102 & 0.997 & 1.22 & 0.056 \\
sST2 & $\mathbf{1 . 5 3 6}$ & $\mathbf{1 . 2 1 6}$ & $\mathbf{1 . 9 9 1}$ & $<\mathbf{0 . 0 0 1}$ \\
TNFR1 & 1.046 & 0.931 & 1.16 & 0.408 \\
cCK18 & 0.723 & 0.101 & 3.097 & 0.702 \\
20S proteasome & $\mathbf{1 . 0 3 7}$ & $\mathbf{1 . 0 0 2}$ & $\mathbf{1 . 0 7 7}$ & $\mathbf{0 . 0 4 4}$ \\
& & & & \\
Multivariable Logistic Regression & & & & \\
Age & $\mathbf{1 . 0 5 9}$ & $\mathbf{1 . 0 2 4}$ & $\mathbf{1 . 1}$ & $\mathbf{0 . 0 0 2}$ \\
sST2 & $\mathbf{1 . 4 5 7}$ & $\mathbf{1 . 1 4 4}$ & $\mathbf{1 . 9 1 1}$ & $\mathbf{0 . 0 0 4}$ \\
20S proteasome & 1.034 & 0.997 & 1.076 & 0.072 \\
\hline
\end{tabular}


Table 4. Univariable and multivariable logistic regression in association with 60-day mortality $(\mathrm{n}=220)$

\begin{tabular}{lllll}
\hline \hline & Odds Ratio & Lower CL & Upper CL & p-Value \\
\hline Univariable Logistic Regression & & & & \\
Age & $\mathbf{1 . 0 5 2}$ & $\mathbf{1 . 0 2 3}$ & $\mathbf{1 . 0 8 7}$ & $<\mathbf{0 . 0 0 1}$ \\
HSP27 & 1.092 & 0.992 & 1.204 & 0.071 \\
sST2 & $\mathbf{1 . 3 3 2}$ & $\mathbf{1 . 0 9 1}$ & $\mathbf{1 . 6 5 9}$ & $\mathbf{0 . 0 0 7}$ \\
TNFR1 & 1.07 & 0.964 & 1.186 & 0.19 \\
cCK18 & 0.566 & 0.081 & 2.42 & 0.502 \\
20S proteasome & 1.032 & 0.997 & 1.07 & 0.076 \\
& & & & \\
Multivariable Logistic-Regression & & & $\mathbf{0 . 0 0 2}$ \\
Age & $\mathbf{1 . 0 5 1}$ & $\mathbf{1 . 0 2}$ & $\mathbf{1 . 0 8 6}$ & $\mathbf{0 . 0 1 6}$ \\
sST2 & $\mathbf{1 . 2 9 8}$ & $\mathbf{1 . 0 6 1}$ & $\mathbf{1 . 6 2 4}$ & \\
\hline
\end{tabular}


Table 5. Univariable and multivariable logistic regression in association with 90 -day mortality $(\mathrm{n}=205)$

\begin{tabular}{lllll}
\hline \hline & Odds Ratio & Lower CL & Upper CL & p-Value \\
\hline Univariable Logistic-Regression & & & & \\
Age & $\mathbf{1 . 0 5 3}$ & $\mathbf{1 . 0 2 2}$ & $\mathbf{1 . 0 8 8}$ & $\mathbf{0 . 0 0 1}$ \\
HSP27 & 1.096 & 0.996 & 1.209 & 0.061 \\
sST2 & $\mathbf{1 . 3 3 5}$ & $\mathbf{1 . 0 9 5}$ & $\mathbf{1 . 6 6 3}$ & $\mathbf{0 . 0 0 7}$ \\
TNFR1 & 1.098 & 0.984 & 1.236 & 0.098 \\
cCK18 & 0.536 & 0.076 & 2.296 & 0.463 \\
20S proteasome & 1.027 & 0.993 & 1.065 & 0.122 \\
& & & \\
Multivariable Logistic-Regression & & & $\mathbf{0 . 0 0 2}$ \\
Age & $\mathbf{1 . 0 5 1}$ & $\mathbf{1 . 0 2}$ & $\mathbf{1 . 0 8 7}$ & $\mathbf{0 . 0 1 4}$ \\
sST2 & $\mathbf{1 . 3 0 4}$ & $\mathbf{1 . 0 6 8}$ & $\mathbf{1 . 6 3 1}$ & \\
\hline
\end{tabular}


Table 6. Univariable and multivariable logistic regression in association with in-hospital mortality $(n=245)$

\begin{tabular}{lllll}
\hline \hline & Odds Ratio & Lower CL & Upper CL & p-Value \\
\hline Univariable Logistic-Regression & & & & \\
Age & $\mathbf{1 . 0 6 2}$ & $\mathbf{1 . 0 2 7}$ & $\mathbf{1 . 1 0 4}$ & $<\mathbf{0 . 0 0 1}$ \\
HSP27 & 1.101 & 0.993 & 1.22 & 0.063 \\
sST2 & $\mathbf{1 . 6 9 7}$ & $\mathbf{1 . 3 1 6}$ & $\mathbf{2 . 2 5 3}$ & $<\mathbf{0 . 0 0 1}$ \\
TNFR1 & 1.079 & 0.963 & 1.2 & 0.16 \\
cCK18 & 0.291 & 0.019 & 1.957 & 0.298 \\
$\mathbf{2 0 S}$ proteasome & $\mathbf{1 . 0 4 4}$ & $\mathbf{1 . 0 0 8}$ & $\mathbf{1 . 0 8 5}$ & $\mathbf{0 . 0 1 9}$ \\
& & & \\
Multivariable Logistic-Regression & & & \\
Age & $\mathbf{1 . 0 6 8}$ & $\mathbf{1 . 0 2 9}$ & $\mathbf{1 . 1 1 4}$ & $\mathbf{0 . 0 0 1}$ \\
sST2 & $\mathbf{1 . 6 3 1}$ & $\mathbf{1 . 2 4 5}$ & $\mathbf{2 . 2 1}$ & $<\mathbf{0 . 0 0 1}$ \\
$\mathbf{2 0 S}$ proteasome & $\mathbf{1 . 0 4 1}$ & $\mathbf{1 . 0 0 3}$ & $\mathbf{1 . 0 8 5}$ & $\mathbf{0 . 0 3 3}$ \\
\hline
\end{tabular}


Table 7. Univariable and multivariable logistic regression in association with requirement of invasive ventilation $(n=245)$

\begin{tabular}{lllll}
\hline \hline & Odds Ratio & Lower CL & Upper CL & p-Value \\
\hline Univariable Cox-Regression & & & & \\
Age & $\mathbf{1 . 0 5 3}$ & $\mathbf{1 . 0 2 1}$ & $\mathbf{1 . 0 9}$ & $\mathbf{0 . 0 0 2}$ \\
HSP27 & $\mathbf{1 . 1 0 8}$ & $\mathbf{1 . 0 0 3}$ & $\mathbf{1 . 2 2 5}$ & $\mathbf{0 . 0 4 1}$ \\
sST2 & $\mathbf{1 . 7 9}$ & $\mathbf{1 . 3 9}$ & $\mathbf{2 . 3 7 3}$ & $<\mathbf{0 . 0 0 1}$ \\
TNFR1 & 1.065 & 0.951 & 1.182 & 0.24 \\
cCK18 & 0.806 & 0.112 & 3.45 & 0.799 \\
20S proteasome & $\mathbf{1 . 0 3 9}$ & $\mathbf{1 . 0 0 4}$ & $\mathbf{1 . 0 7 9}$ & $\mathbf{0 . 0 3 1}$ \\
& & & & \\
Multivariable Cox-Regression & & & & \\
Age & $\mathbf{1 . 0 5 5}$ & $\mathbf{1 . 0 2}$ & $\mathbf{1 . 0 9 6}$ & $\mathbf{0 . 0 0 3}$ \\
HSP27 & 1.028 & 0.915 & 1.155 & 0.635 \\
sST2 & $\mathbf{1 . 7 0 9}$ & $\mathbf{1 . 3 0 6}$ & $\mathbf{2 . 3 1}$ & $<\mathbf{0 . 0 0 1}$ \\
20S proteasome & 1.032 & 0.993 & 1.074 & 0.094 \\
\hline
\end{tabular}


Table 8. Univariable and multivariable logistic regression in association with requirement of oxygen therapy $(n=245)$

\begin{tabular}{lllll}
\hline \hline & Odds Ratio & Lower CL & Upper CL & p-Value \\
\hline Univariable Logistic-Regression & & & & \\
Age & 0.995 & 0.974 & 1.016 & 0.662 \\
HSP27 & $\mathbf{1 . 2 1 8}$ & $\mathbf{1 . 0 9 1}$ & $\mathbf{1 . 3 7 4}$ & $<\mathbf{0 . 0 0 1}$ \\
SST2 & $\mathbf{1 . 2 5 8}$ & $\mathbf{1 . 0 8 2}$ & $\mathbf{1 . 4 7 5}$ & $\mathbf{0 . 0 0 3}$ \\
TNFR1 & 1.107 & 0.98 & 1.289 & 0.146 \\
cCK18 & 0.747 & 0.213 & 2.985 & 0.651 \\
20S proteasome & $\mathbf{1 . 1 7 7}$ & $\mathbf{1 . 0 8 4}$ & $\mathbf{1 . 3}$ & $<\mathbf{0 . 0 0 1}$ \\
& & & & \\
Multivariable Logistic Regression & & & & \\
HSP27 & $\mathbf{1 . 1 3 4}$ & $\mathbf{1 . 0 1 2}$ & $\mathbf{1 . 2 8 2}$ & $\mathbf{0 . 0 3 7}$ \\
sST2 & $\mathbf{1 . 1 7 5}$ & $\mathbf{1 . 0 0 3}$ & $\mathbf{1 . 3 8}$ & $\mathbf{0 . 0 4 6}$ \\
20S proteasome & $\mathbf{1 . 1 4 7}$ & $\mathbf{1 . 0 5 4}$ & $\mathbf{1 . 2 6 9}$ & $\mathbf{0 . 0 0 4}$ \\
\hline
\end{tabular}


Table 9: Correlations between the investigated inflammatory markers, based on Spearman's rank correlation analysis

\begin{tabular}{lll}
\hline Serum markers & Rank correlation $(\mathrm{R})$ & p-value \\
\hline sST2 vs. 20 S proteasome & $\mathbf{0 . 1 9 0}$ & $\mathbf{0 . 0 0 3}$ \\
sST2 vs. HSP27 & $\mathbf{0 . 2 3 7}$ & $<\mathbf{0 . 0 0 1}$ \\
sST2 vs. cCK18 & -0.039 & n.s. \\
sST2 vs. TNFR1 & $\mathbf{0 . 1 7 6}$ & $\mathbf{0 . 0 0 6}$ \\
& $\mathbf{0 . 2 8 0}$ & $<\mathbf{0 . 0 0 1}$ \\
20S proteasome vs. HSP27 & -0.024 & n.s. \\
20S proteasome vs. cCK18 & 0.005 & n.s. \\
20S proteasome vs. TNFR1 & 0.011 & n.s. \\
HSP27 vs. cCK18 & 0.038 & n.s. \\
HSP27 vs. TNFR1 & -0.021 & n.s. \\
cCK18 vs. TNFR1 & & \\
& & \\
\hline
\end{tabular}




\section{Supplementary material}

The following are available in the supplementary file: Figure S1 ROC Curves for the endpoint 30-day mortality of investigated parameters; Figure S2 ROC Curves for the endpoint 60-day mortality of investigated parameters, Figure S3 ROC Curves for the endpoint 90-day mortality of investigated parameters, Figure S4 ROC Curves for the endpoint in-hospital mortality of investigated parameters, Table S1 AUC of ROC curves for the endpoint 30day mortality of investigated parameters, Table S2 AUC of ROC curves for the endpoint 60-day mortality of investigated parameters, Table S3 AUC of ROC curves for the endpoint 90-day mortality of investigated parameters, Table S4 AUC of ROC curves for the endpoint in-hospital mortality of investigated parameters Authors' contributions

HJA: conceptualization; RW, MTL, and HJA: design and manuscript writing; SK and CL: patient care; RW: serum sample collection; RW, MTL, SK, CL, JB, HJAL, PK, AG, DT: manuscript revision. PK, AG: statistical analyses Acknowledgement: This study was performed as part of a Doctoral thesis (MTL).

\section{Funding}

This study was funded by FOLAB (Clinic of Thoracic Surgery). MTL and H.J.A. were supported by the Vienna Business Agency “APOSEC to clinic,” (ID 2343727, 2018-2020), and by the Aposcience AG under group leader H.J.A.

Institutional Review Board Statement

The study was approved by the Ethics Committee of the German-Saxonian Board of Physicians, Dresden, Germany (EK-BR-88/20.1), and it was conducted in accordance with the current version of the Declaration of Helsinki.

\section{Informed Consent Statement}

All patients provided written informed consent prior to blood collection and participation.

Data Availability Statement:

All data and materials support our published claims and comply with field standards. All data are available upon request.

\section{Acknowledgements}

This research project was financed in part by the FFG Grant "APOSEC" (852748 and 862068; 2015-2019), by the Vienna Business Agency “APOSEC to clinic,” (ID 2343727, 2018-2020), and by the Aposcience AG under group leader HJA. MM was funded by the Sparkling Science Program of the Austrian Federal Ministry of Education, Science and Research (SPA06/055).

\section{Consent for publication}

All authors read the manuscript and approved publication.

Conflicts of interest/Competing interests

The authors declare no competing interests. 


\section{References}

[1] B. Salzberger, F. Buder, B. Lampl, B. Ehrenstein, F. Hitzenbichler, and F. Hanses, "Epidemiology of SARS-CoV-2 infection and COVID-19,” Internist, vol. 61, no. 8, pp. 782-788, Aug. 2020, doi: 10.1007/s00108-020-00834-9.

[2] R. Koch-Institut, "Epidemiologisches Bulletin Höhere Letalität, längere Beatmungsdauer bei COVID-19 im Vergleich zu schwerer Influenza,” 2020, Accessed: Jul. 13, 2021. [Online]. Available: www.rki.de/epidbull

[3] S. de Biasi et al., "Marked T cell activation, senescence, exhaustion and skewing towards TH17 in patients with COVID-19 pneumonia," Nature Communications, vol. 11, no. 1, Dec. 2020, doi: 10.1038/s41467-020-17292-4.

[4] G. Chen et al., "Clinical and immunological features of severe and moderate coronavirus disease 2019," Journal of Clinical Investigation, vol. 130, no. 5, pp. 2620-2629, May 2020, doi: 10.1172/JCI137244.

[5] S. F. Pedersen and Y. C. Ho, "SARS-CoV-2: A storm is raging," Journal of Clinical Investigation, vol. 130, no. 5. American Society for Clinical Investigation, pp. 2202-2205, May 01, 2020. doi: 10.1172/JCI137647.

[6] Z. Xu et al., "Pathological findings of COVID-19 associated with acute respiratory distress syndrome," The Lancet Respiratory Medicine, vol. 8, no. 4, pp. 420-422, Apr. 2020, doi: 10.1016/S22132600(20)30076-X.

[7] M. F. Osuchowski et al., "The COVID-19 puzzle: deciphering pathophysiology and phenotypes of a new disease entity," The Lancet Respiratory Medicine, vol. 9, no. 6, pp. 622-642, Jun. 2021, doi: 10.1016/s2213-2600(21)00218-6.

[8] Y. Zhang, Y. Chen, and Z. Meng, "Immunomodulation for Severe COVID-19 Pneumonia: The State of the Art," Frontiers in Immunology, vol. 11. Frontiers Media S.A., Nov. 09, 2020. doi: 10.3389/fimmu.2020.577442.

[9] R. J. Jose and A. Manuel, "COVID-19 cytokine storm: the interplay between inflammation and coagulation," The Lancet Respiratory Medicine, vol. 8, no. 6. Lancet Publishing Group, pp. e46-e47, Jun. 01, 2020. doi: 10.1016/S2213-2600(20)30216-2.

[10] M. Tian et al., "Hsp-27 levels and thrombus burden relate to clinical outcomes in patients with STsegment elevation myocardial infarction," Oncotarget, vol. 8, no. 43, pp. 73733-73744, 2017, doi: 10.18632/oncotarget. 17852.

[11] N. Tang, D. Li, X. Wang, and Z. Sun, "Abnormal coagulation parameters are associated with poor prognosis in patients with novel coronavirus pneumonia," Journal of Thrombosis and Haemostasis, vol. 18, no. 4, pp. 844-847, Apr. 2020, doi: 10.1111/jth.14768.

[12] F. Zhou et al., "Clinical course and risk factors for mortality of adult inpatients with COVID-19 in Wuhan, China: a retrospective cohort study," The Lancet, vol. 395, no. 10229, pp. 1054-1062, Mar. 2020, doi: 10.1016/S0140-6736(20)30566-3. 
[13] R. J. Jose and A. Manuel, "COVID-19 cytokine storm: the interplay between inflammation and coagulation," The Lancet Respiratory Medicine, vol. 8, no. 6. Lancet Publishing Group, pp. e46-e47, Jun. 01, 2020. doi: 10.1016/S2213-2600(20)30216-2.

[14] L. Gattinoni et al., "COVID-19 pneumonia: different respiratory treatments for different phenotypes?," Intensive Care Medicine, vol. 46, no. 6, p. 1099, Jun. 2020, doi: 10.1007/S00134-020-06033-2.

[15] L. Gattinoni, D. Chiumello, and S. Rossi, “COVID-19 pneumonia: ARDS or not?,” Critical Care, vol. 24, no. 1, Apr. 2020, doi: 10.1186/S13054-020-02880-Z.

[16] A. Mazzoni et al., "Impaired immune cell cytotoxicity in severe COVID-19 is IL-6 dependent," Journal of Clinical Investigation, vol. 130, no. 9, pp. 4694-4703, Sep. 2020, doi: 10.1172/JCI138554.

[17] R. S. Hotchkiss, G. Monneret, and D. Payen, "Sepsis-induced immunosuppression: From cellular dysfunctions to immunotherapy," Nature Reviews Immunology, vol. 13, no. 12. Nat Rev Immunol, pp. 862-874, Dec. 2013. doi: 10.1038/nri3552.

[18] H. Wang et al., “Overview: Systemic Inflammatory Response Derived From Lung Injury Caused by SARS-CoV-2 Infection Explains Severe Outcomes in COVID-19," Frontiers in Immunology | www.frontiersin.org, vol. 1, p. 1626, 2020, doi: 10.3389/fimmu.2020.01626.

[19] G. Roth et al., "Susceptibility to programmed cell death in T-lymphocytes from septic patients: A mechanism for lymphopenia and Th2 predominance," Biochemical and Biophysical Research Communications, vol. 308, no. 4, pp. 840-846, Sep. 2003, doi: 10.1016/S0006-291X(03)01482-7.

[20] G. A. Roth et al., "Heightened levels of circulating 20 S proteasome in critically ill patients," European Journal of Clinical Investigation, vol. 35, no. 6, pp. 399-403, Jun. 2005, doi: 10.1111/j.13652362.2005.01508.x.

[21] G. A. Roth et al., "Elevated serum levels of epithelial cell apoptosis-specific cytokeratin 18 neoepitope M30 in critically ill patients," Shock, vol. 22, no. 3, pp. 218-220, Sep. 2004, doi: 10.1097/01.shk.0000136098.49672.0e.

[22] M. Brunner et al., "Increased levels of soluble ST2 protein and IgG1production in patients with sepsis and trauma," Intensive Care Medicine, vol. 30, no. 7, pp. 1468-1473, 2004, doi: 10.1007/s00134-0042184-X.

[23] M. Mildner et al., "Primary sources and immunological prerequisites for sST2 secretion in humans," Cardiovascular Research, vol. 87, no. 4, pp. 769-777, Sep. 2010, doi: 10.1093/cvr/cvq104.

[24] S. Hacker et al., "Elevated HSP27, HSP70 and HSP90 $\alpha$ in chronic obstructive pulmonary disease: Markers for immune activation and tissue destruction," Clinical Laboratory, vol. 55, no. 1-2, pp. 31-40, 2009, Accessed: Jun. 20, 2021. [Online]. Available: https://pubmed.ncbi.nlm.nih.gov/19350847/

[25] H. J. Ankersmit et al., "Increased serum levels of HSP27 as a marker for incipient chronic obstructive pulmonary disease in young smokers," Respiration, vol. 83, no. 5, pp. 391-399, May 2012, doi: $10.1159 / 000336557$. 
[26] M. Zimmermann et al., "Circulating heat shock protein 27 as a biomarker for the differentiation of patients with lung cancer and healthy controls - A clinical comparison of different enzyme linked immunosorbent assays," Clinical Laboratory, vol. 60, no. 6, pp. 999-1006, 2014, doi: 10.7754/Clin.Lab.2013.130526.

[27] D. Traxler et al., "The inflammatory markers sST2, HSP27 and hsCRP as a prognostic biomarker panel in chronic heart failure patients," Clinica Chimica Acta, vol. 510, pp. 507-514, Nov. 2020, doi: 10.1016/j.cca.2020.07.050.

[28] M P Leers et al., "Immunocytochemical detection and mapping of a cytokeratin 18 neo-epitope exposed during early apoptosis - Leers - 1999 - The Journal of Pathology - Wiley Online Library,” 1999. https://onlinelibrary.wiley.com/doi/10.1002/(SICI)1096-9896(199904)187:5\%3C567::AIDPATH288\%3E3.0.CO;2-J (accessed Jul. 13, 2021).

[29] “WHO R\&D Blueprint novel Coronavirus COVID-19 Therapeutic Trial Synopsis,” 2020.

[30] R.-S. Miftode et al., "The Novel Perspectives Opened by ST2 in the Pandemic: A Review of Its Role in the Diagnosis and Prognosis of Patients with Heart Failure and COVID-19," Diagnostics, vol. 11, no. 2 , p. 175, Jan. 2021, doi: 10.3390/diagnostics11020175.

[31] M. S. Abers et al., "An immune-based biomarker signature is associated with mortality in COVID-19 patients,” JCI Insight, vol. 6, no. 1, Jan. 2021, doi: 10.1172/jci.insight.144455.

[32] Z. Zeng et al., "Serum-soluble ST2 as a novel biomarker reflecting inflammatory status and illness severity in patients with COVID-19," Biomarkers in Medicine, vol. 14, no. 17, pp. 1619-1629, Dec. 2020, doi: 10.2217/bmm-2020-0410.

[33] D. A. Pascual-Figal and J. L. Januzzi, “The biology of ST2: The international ST2 consensus panel,” American Journal of Cardiology, vol. 115, no. 7, pp. 3B-7B, Apr. 2015, doi: 10.1016/j.amjcard.2015.01.034.

[34] S. Hacker et al., "Increased serum concentrations of soluble ST2 predict mortality after burn injury," Clinical Chemistry and Laboratory Medicine, vol. 56, no. 12, pp. 2079-2087, Dec. 2018, doi: 10.1515/cclm-2018-0042.

[35] S. Kumar, M. N. Tzimas, D. E. Griswold, and P. R. Young, "Expression of ST2, an interleukin-1 receptor homologue, is induced by proinflammatory stimuli," Biochemical and Biophysical Research Communications, vol. 235, no. 3, pp. 474-478, Jun. 1997, doi: 10.1006/bbrc.1997.6810.

[36] M. Brunner et al., "Increased levels of soluble ST2 protein and IgG1 production in patients with sepsis and trauma," Intensive Care Med, vol. 30, pp. 1468-1473, 2004, doi: 10.1007/s00134-004-2184-x.

[37] P. Opfermann et al., "Early sST2 Liberation after Implantation of a Left Ventricular Assist Device in Patients with Advanced Heart Failure," Journal of Immunology Research, vol. 2020, 2020, doi: $10.1155 / 2020 / 5826176$. 
[38] M. S. Abers et al., "An immune-based biomarker signature is associated with mortality in COVID-19 patients European Reference Network on Haematological Diseases, and European Reference Network on Hereditary Metabolic,” vol. 19, doi: 10.1172/jci.

[39] S. K. Calderwood, J. Gong, and A. Murshid, "Extracellular HSPs: The complicated roles of extracellular HSPs in immunity," Frontiers in Immunology, vol. 7, no. APR. Frontiers Media S.A., Apr. 25, 2016. doi: 10.3389/fimmu.2016.00159.

[40] S. Salari et al., "Extracellular HSP27 acts as a signaling molecule to activate NF- $\kappa \mathrm{B}$ in macrophages," Cell Stress and Chaperones, vol. 18, no. 1, pp. 53-63, Jan. 2013, doi: 10.1007/s12192-012-0356-0.

[41] T. Haider et al., "Systemic release of heat-shock protein 27 and 70 following severe trauma," Scientific Reports, vol. 9, no. 1, Dec. 2019, doi: 10.1038/s41598-019-46034-w.

[42] R. Polanowska-Grabowska and A. R. L. Gear, "Heat-shock proteins and platelet function," Platelets, vol. 11, no. 1. Carfax Publishing Company, pp. 6-22, 2000. doi: 10.1080/09537100075742.

[43] M. Zimmermann et al., "Heat shock protein 27 as a predictor of prognosis in patients admitted to hospital with acute COPD exacerbation," Cell Stress and Chaperones, vol. 25, no. 1, pp. 141-149, Jan. 2020, doi: 10.1007/s12192-019-01057-0.

[44] D. Traxler et al., "Fractional heat shock protein 27 urine excretion as a short-term predictor in acute exacerbation of chronic obstructive pulmonary disease," Annals of Translational Medicine, vol. 9, no. 2, pp. 117-117, Jan. 2021, doi: 10.21037/ATM-20-3683).

[45] S. X. Gu et al., "Thrombocytopathy and endotheliopathy: crucial contributors to COVID-19 thromboinflammation," Nature Reviews. Cardiology, vol. 18, no. 3, p. 1, Mar. 2021, doi: 10.1038/S41569-020-00469-1.

[46] M. Zimmermann et al., "Discrimination of clinical stages in non-small cell lung cancer patients by serum HSP27 and HSP70: A multi-institutional case-control study," Clinica Chimica Acta, vol. 413, no. 13-14, pp. 1115-1120, Jul. 2012, doi: 10.1016/j.cca.2012.03.008.

[47] "Circulating 20S Proteasome in Patients with Non-metastasized Breast Cancer | Anticancer Research." https://ar.iiarjournals.org/content/31/6/2197.long (accessed Aug. 12, 2021).

[48] M. Majetschak et al., "Circulating Proteasomes After Burn Injury,” Journal of burn care \& research : official publication of the American Burn Association, vol. 31, no. 2, p. 243, Mar. 2010, doi: 10.1097/BCR.0B013E3181D0F55D.

[49] K. Egerer et al., "Circulating proteasomes are markers of cell damage and immunologic activity in autoimmune diseases.," The Journal of Rheumatology, vol. 29, no. 10, 2002.

[50] V. Dwivedi, K. Yaniv, and M. Sharon, "Beyond cells: The extracellular circulating 20S proteasomes," Biochimica et Biophysica Acta (BBA) - Molecular Basis of Disease, vol. 1867, no. 3, p. 166041, Mar. 2021, doi: 10.1016/J.BBADIS.2020.166041. 
